【研究简报】

\title{
人正常骨髓 $\mathrm{CD34}^{+}$造血干/祖细胞 的超微结构
}

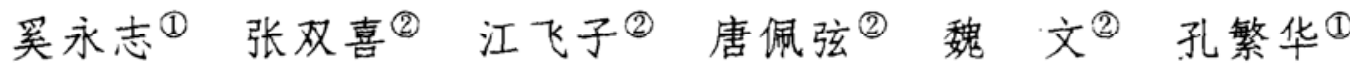

(1)军事医学科学院 307 医院移植免废中心, 北京 100039 ; (2)军事医学科学院基础医学研究所, 北京 100850)

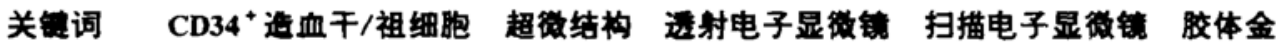

时至今日, 人类究竟有无造血干细胞 (HSC) 已不再是争论的焦点, 对该问题的答案是肯定 的. 目前所认识 HSC 的某些特性已远非简单的高度自我更新与多向分化这一定义就能概括 其全貌. 此概念仅基于细胞的功能, 而无任何形态学的特征. 并且在 80 年代中期致力于造血 干/祖细胞 (HSC/HPC) 形态学或本质的探索还被认为是“浪费时间”, 喻作恰似以生化或电子 显微镜手段是不可能证明细胞因子的生物活性一样. 其主要障碍在于: (1)HSC 在人体内含量

$90.8 \%{ }^{1)}$. 逆转病毒载体具有高效感染, 高效表达, 对宿主细胞无损伤的特点, 能够感染多层 细胞的深部和三维培养肿瘤细胞球的内部, 因此它常被用来作为基因的表达载体. 本研究在 注入 $\mathrm{pMR}$ 病毒的一些裸鼠形成可见的较小的瘤结节, 这可能是由于一些 GAOK 细胞没有被 pMR 感染而形成肿瘤结节. 人肿瘤细胞的良好生长和浸润与其所在的微环境密切相关, 原位 移植使肿瘦具有与人体肿瘦相似的自然状态, 可以使肿瘤生长较好并基本保持原有特征 ${ }^{[6]}$, 是理想的研究模型. 本研究利用裸鼠原位移植模型, 模拟病人体内状态, 采用气管注入病毒上 清, 用抗癌药对耐药肺癌 GAOK 细胞进行治疗, 使肿㿔成瘤率明显降低, 肿瘤体积明显减小, 说明本方法即基因-药物法治疗 NSCLC 行之有效, 这对临床耐药肺癌的治疗具有重要的理论 和实用价值. 至于气管注入 MDR1 ribozyme 逆转病毒 pMR 是否对造血系统有无影响, 我们 正在进一步研究.

\section{參考文献}

1 高掁强, 高志萍. 多向耐药基因研究进展. 中华病理学杂志, 1996,3:185 187

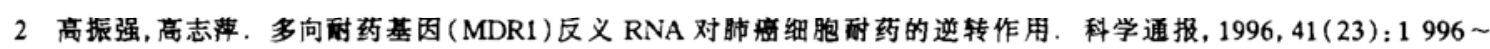
2000

3 高振强, 张志华, 高志莎. 人肺癌多向解药基因表达及其临休意义. 中华内科杂志, 1996, 7:482 483

4 Symons R. Self-cleavage of RNA in the replicative of small pathogens of plants and animals. TIBS, 1989,$14 ; 445 \sim 448$

5 Kobayashi H, Dorai T, Holland J, et al. Reversal of drug sensitivity in multidrug-resistant tumor cells by an MDR1 (PGY1) ribozyme. Cancer Res, 1994, 53: $1271 \sim 1275$

6 McLemore T L, Liu M C, Blacker P C, et al. Novel intrapulmonary model for orthotopic propagations in human lung cancers in athymic nude mice. Cancer Res, 1987, 47: 5 132 5 140

（1996-05-16 收稿, 1996-09-03 收修改稿)

1）见529页脚注 
甚微, 骨榷 $<0.01 \% \sim 1 \%$, 外周血 $<0.001 \% \sim 0.1 \%$; (2)缺乏特异的物化及形态学特征; (3)无 直接测定人类 HSC 的体内实验方法; (4)细胞分离富集技术的落伍; (5)相关科学发展的局限性. 从而严重阻碍了对人类 HSC 的深入研究, 以致于今天对于 HSC/HPC 的认识、概念与探索仍 局限于功能方面 ${ }^{[1,2]}$.

近年来, 由于新学科、边缘与交叉学科在实验血液学中的充分渗透; 大量造血细胞分化抗 原单克隆抗体尤其是抗 CD34 单抗的问世; 体内外实验与临床造血细胞移植的结果确丵 CD34 抗原分子选择性地在人类 HSC/HPC 上的表达; 多维流式细胞仪分析的广泛应用; 检测人类各 种造血祖细胞方法的建立; 以及发现与重组了十几种早晚期造血生长因子, 使人们已有能力获 取高度纯一的 $\mathrm{CD} 34^{+} \mathrm{HSC} / \mathrm{HPC}$ 及其亚群,进行各种相关生物学特性的研究. HSC/HPC 究竟 有无形态特征, 其本质如何, 超微结构又是怎样, 这些至今在国际上仍是一个谜 ${ }^{[3.4]}$.

应用阳性选择策略, 我们以往的工作建立了免疫磁球分离术, 已成功地高纯度富集了人骨 顝 $\mathrm{CD} 34^{+} \mathrm{HSC} / \mathrm{HPC}$, 并对其相关亚群作了较全面的分析 ${ }^{[5,6]}$. 为了观察 $\mathrm{CD} 34^{+} \mathrm{HSC} / \mathrm{HPC}$ 的形态与超微结构, 新近我们结合免疫磁球-流式细胞分选两步法, 以充分提高 $\mathrm{CD}^{2} 4^{+} \mathrm{HSC} /$ $\mathrm{HPC}$ 的纯度,使其阳性率达到 $99 \% \sim 100 \%$, 分别进行了扫描及免疫胶体金标记后的透射电子 显微镜超微研究.

\section{1 材料与方法}

\section{1 骨螴细胞来源与制备}

取胸外科无血液疾患切除的助骨, 无菌下用比重 $1.077 \mathrm{~g} / \mathrm{mL}$ 的 Ficoll-Hypaque 分出单个 核细胞, 经 RPMI1640/HSA(1\%)洗涤 2 次后备用.

\subsection{Isolex ${ }^{\mathrm{TM}} 50$ 或 CIMS-100 系统亩集 $\mathrm{CD}^{2} 4^{+}$HSC/HPC}

应用阳性选择策略, 采用我们已建立的免度磁球分离方法 ${ }^{[5]}$.

\section{3 流式细胞仪二次分选 $\mathrm{CD34}^{+} \mathrm{HSC} / \mathrm{HPC}$}

参照我们已建立方法, 用FACS 440 (BD公司) 对 Isolex ${ }^{\mathrm{TM}} 50$ 或 CIMS-100系统富集的 $\mathrm{CD} 34^{+} \mathrm{HSC} / \mathrm{HPC}$ 进行低速 2 次分选, 根据低-中度前向角和极低-低度垂直角光散射信号建立 窗口进行分离.

\section{$1.4 \mathrm{CD}^{+} 4^{+} \mathrm{HSC} / \mathrm{HPC}$ 纯度鉴定}

方法见文献[5], 采用间接免疫荧光标记, 抗 CD34 单抗(Tuk3) 与 FITC-F ( $\left.a b^{\prime}\right)_{2}$ 羊抗鼠二 抗购自 Dako 公司,标记后在 FACS440 上检测.

\section{5 扫描电子显微镜 (SEM) 样品制备}

$\mathrm{CD} 34^{+} \mathrm{HSC} / \mathrm{HPC}$ 细胞悬液滴在涂有血清的盖玻片上静止 $10 \mathrm{~min}$, 用 $3 \%$ 戊二醛固定 30 $\mathrm{min}$, 然后用 $1 / 15 \mathrm{~mol} / \mathrm{L}$ PBS 洗 $10 \mathrm{~min}$, 再用 $1 \%$ 锇酸固定 $30 \mathrm{~min}, 1 / 15 \mathrm{~mol} / \mathrm{L}$ PBS 洗 $10 \mathrm{~min}$. 乙醇梯度脱水, 以六甲基二硅胺干燥样品, 离了泍射喷涂白金, S-505 扫描电子显钽镜观察照 相.

\section{6 遁射电子显微镜( TEM) 样品制备}

采用特异性免疫胶体金标记, 方法参照文献[6]. 将 IGSS 标记的 CD $34^{+} \mathrm{HSC} / \mathrm{HPC}$ 离心 成团, $3 \%$ 戊二醛固定 $2 \mathrm{~h}, 1 / 15 \mathrm{~mol} / \mathrm{L}$ PBS 水洗 $2 \mathrm{~h}$. 再用 $1 \%$ 锇酸固定 $2 \mathrm{~h}, 1 / 15 \mathrm{~mol} / \mathrm{L} \mathrm{PBS}$ 水洗后, 乙醇-丙酮梯度脱水, Epon 812 环氧树脂包埋, ULTRACUTE 超薄切片. 所切薄片用 醋酸钠、柠檬酸铅双重染色后, EM-400T 透射电子显微镜观察照相. 


\section{2 结果与讨论}

目前国际上对于 $\mathrm{CD} 34^{+} \mathrm{HSC} / \mathrm{HPC}$ 的认识和研究主要局限于其功能方面, 有关 $\mathrm{CD} 34^{+}$ $\mathrm{HSC} / \mathrm{HPC}$ 形态学的探索胗胗无几, 而揭示其超微结构特征的研究未见任何报道 ${ }^{[1,3]}$, 本文是 当今首次较系统分析人类 $\mathrm{CD} 34^{+} \mathrm{HSC} / \mathrm{HPC}$ 超微结构的研究. 为较全面准确地评价, 我们根 据阳性选择策略, 选用最有效的富集手段设计组合了 Isolex $^{\mathrm{TM}} 50-\mathrm{FACS} 4402$ 次分选 ${ }^{[7]}$, 从而 保证了所获取的 CD $34^{+} \mathrm{HSC} / \mathrm{HPC}$ 纯度达到 $100 \%$, 见图 1(a), (b).

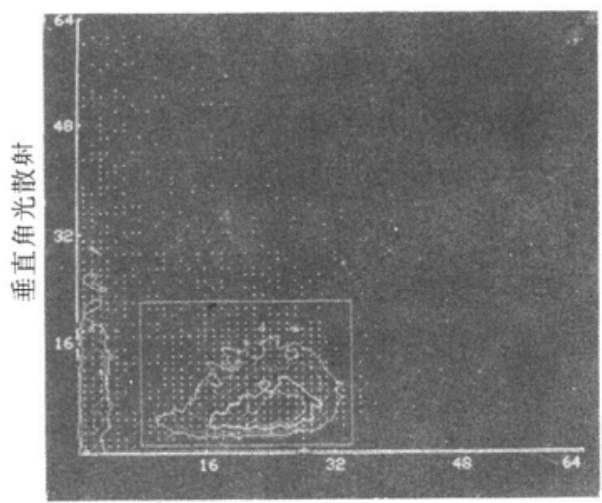

前向角光散射

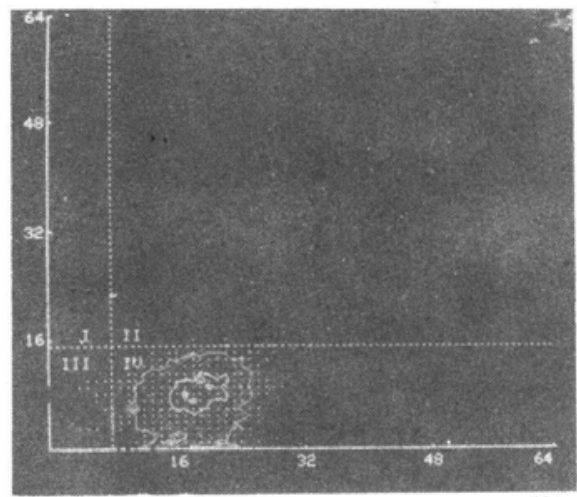

CD34-FITC (b)

图 1 流式细胞仪分析两步法富集的高纯度骨髓 CD34 ${ }^{+} \mathrm{HSC} / \mathrm{HPC}$

的光散射性及 $\mathrm{CD} 34^{+} \mathrm{HSC} / \mathrm{HPC}$ 的含量

(a) 示 $\mathrm{CD} 34^{+} \mathrm{HSC} / \mathrm{HPC}$ 的前向角及垂直角光散射; (b) 示富集的 CD $34^{+} \mathrm{HSC} / \mathrm{HPC}$ 的纯度为 $100 \%$ (IV区)

在光学显微镜下, 根据 $\mathrm{CD} 34^{+} \mathrm{HSC} / \mathrm{HPC}$ 细胞大小、核浆比例、核仁有无、核染色质等结 构性质, 我们已将 $\mathrm{CD} 34^{+} \mathrm{HSC} / \mathrm{HPC}$ 划分为 3 型. 从胞体大小讲, I 型胞体略大于淋巴细胞, II 型胞体为典型的小淋巴细胞状, III 型细胞胞体大小极不均一. 然而这远不足以深入地认识 $\mathrm{CD} 34^{+} \mathrm{HSC} / \mathrm{HPC}$ 细胞的内部结构, 因此只有通过电子显微镜手段, 以其粒子较小、分辨率高 和放大倍数大等特点, 能从超微结构形态学方面更加深入地了解 $\mathrm{CD} 34^{+} \mathrm{HSC} / \mathrm{HPC}$ 的本质. 在扫描电子显微镜下, 仅根据细胞体积的不同亦可明确地将 $\mathrm{CD} 34^{+} \mathrm{HSC} / \mathrm{HPC}$ 划分为大中小 3 型, 见图 2(a). 小型细胞呈球状, 表面可见不等量的短棒状结构及迭起的皱膜; 中型细胞呈
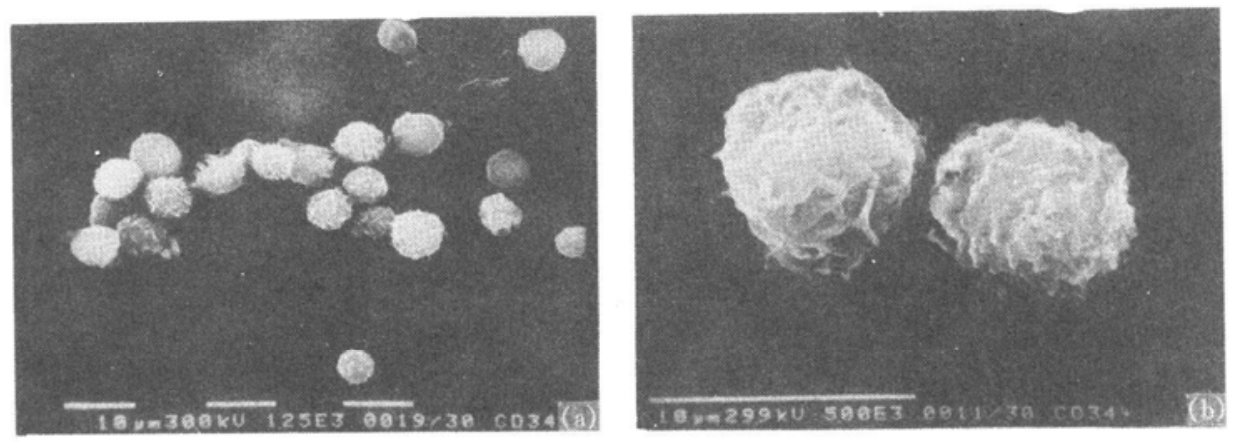

图 2 高纯度人骨爴 $\mathrm{CD} 34^{+} \mathrm{HSC} / \mathrm{HPC}$ 扫描电子显微镜的超微结构 (a)示不同大小的 $\mathrm{CD}_{34}{ }^{+} \mathrm{HSC} / \mathrm{HPC}$; (b) 示中型 $\mathrm{CD} 34^{+} \mathrm{HSC} / \mathrm{HPC}$ 的放大后观察 
圆或椭圆形, 较小型细胞略大, 表面可见数根线毛或微线毛, 哙样与颗粒状突起交错陡峭; 大型 细胞较圆, 表面相对光滑, 皱纹或颗粒状突起较少. 图 2(b) 为中型细胞的放大观察.

在透射电子显微镜下, 我们以 $\mathrm{CD} 34$ 单克隆抗体-胶体金探针标记 $\mathrm{CD} 34$ 抗原, 由于金颗粒 圆, 电子密度高, 界限清晰, 很容易识别, 从而充分保证所观察的造血细胞是 CD $34^{+} \mathrm{HSC} /$ HPC. 从被标记有特异性金颗粒的细胞看, CD $34^{+} \mathrm{HSC} / \mathrm{HPC}$ 亦呈多种形式. 其一, 细胞呈长 椭圆、不规则并有凹陷或指状突起, 有的带有少量线毛, 核呈圆形, 约占胞体的 $80 \% \sim 90 \%$, 核 仁大而明显, 常染色质居中占绝大部分, 常通向核膜孔, 异染质很少, 分布于核周围, 核膜孔较 多, 胞浆量甚少, 核浆之比最大, 核糖体较丰富, 线粒体一般较多, 内质网可见, 这雷同于光学显 微镜下所描述的拟定 I 型候选干细胞, 见图 3(a) (c). 其二细胞大小不一, 胞体呈圆或椭圆 形, 表面相对光滑或有少量突起, 核呈圆或卵圆形带有轻凹, 偏向胞体一侧, 核仁少, 异染质较 前者有所增加, 常质减少, 核膜孔增多, 核浆之比减少, 胞浆量增多, 线粒体明显增加, 游离核糖 体较多呈颗粒状, 常可见内质网, 该群细胞类似于光学显微镜下所描述的 III 型细胞, 已趋于定 向某一祖系细胞阶段.
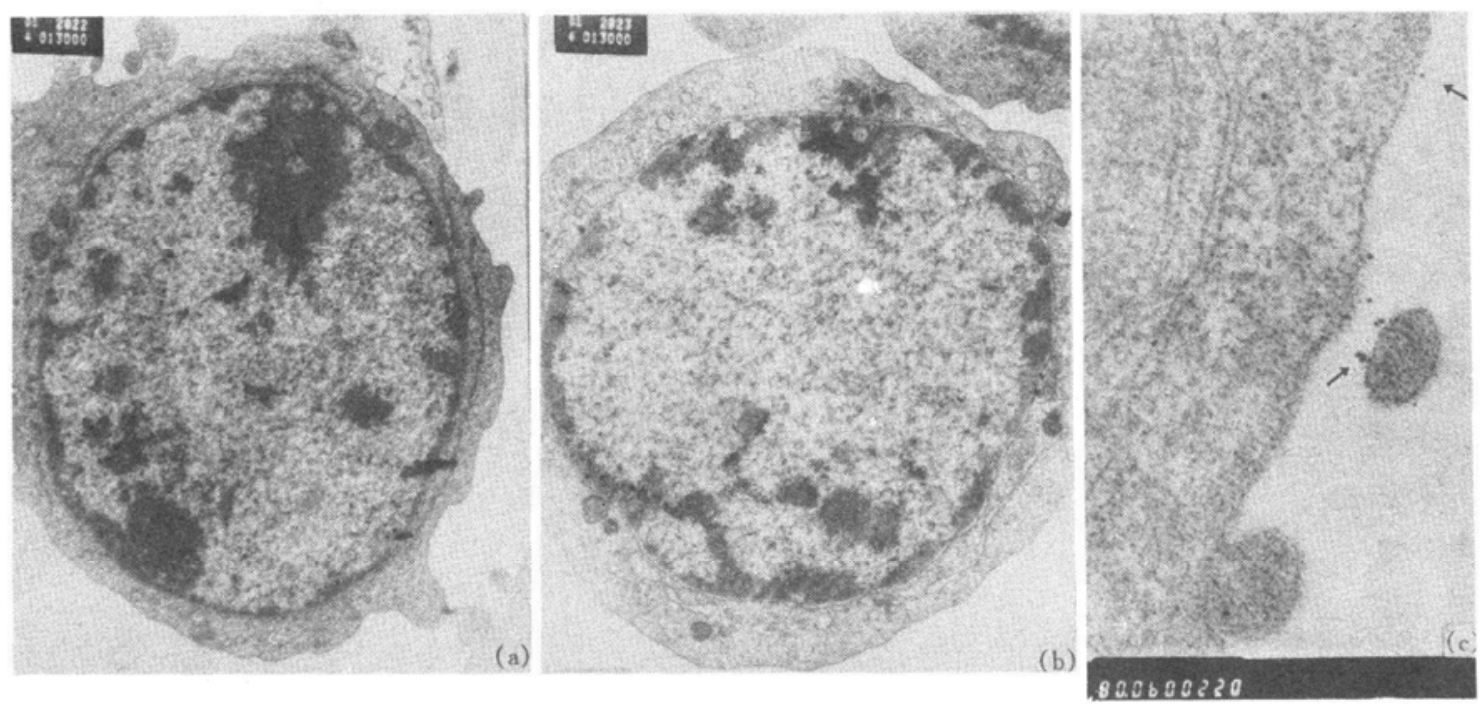

图 3 特异性免疫胶体金标记 $\mathrm{CD} 34$ 抗原后透射电子显微镜下的人骨髓 $\mathrm{CD} 34^{+} \mathrm{HSC} / \mathrm{HPC}$ 超微结构 (a)，(b) 分示拟定的 CD $34^{+}$候选干细胞; (c) 中的箭头所示处为标记的 $5 \mathrm{~nm}$ 金颗粒

从上述超微结构的分析, 人类 $\mathrm{CD} 34^{+} \mathrm{HSC} / \mathrm{HPC}$ 从胞体大小看是极不均一的, 呈多态性, 无论是扫描抑或透射电子显微镜以及光学显微镜下均证实此点, 此结果与细胞化学以及亚群 的分析是一致的 ${ }^{[6]}$. 因此, 对 $\mathrm{CD} 34^{+} \mathrm{HSC} / \mathrm{HPC}$ 本质或形态学的认识应是多方面多层次的, 更深更细的探索应是区分各功能亚群的形态与超微特征, 如采用针对各分化抗原的 CD 单抗 交联不同粒径的金颗粒进行双重或多重标记.

致谢 本工作为国家自然科学基金(批准号:39370272)与美国 Baxter 公司(931108)资助项目.

\section{参考文献}

1 奚永志. 人类 $\mathrm{CD} 34^{+}$造血干/祖细胞的分子生物学特性. 生理科学进展, 1995, 26:19 
【研究简报】

\section{光系统 II 反应中心电荷分离和 能量传递的动力学研究}

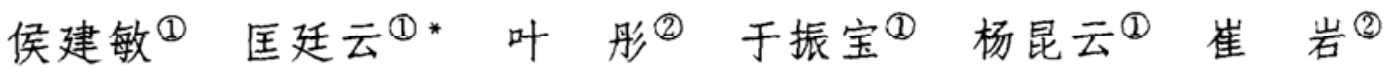

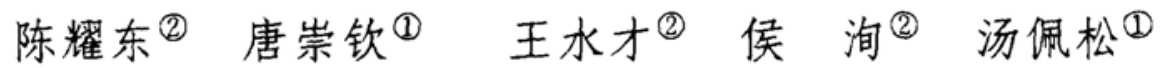

(1)中国科学院植物研究所光合作用实验室, 北京 100093; (2)中国科学院西安光学棈密机械研究所瞹态学技术 国家重点实验室,西安 710068. *联系人)

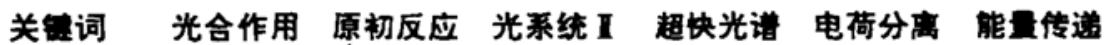

光合作用中最核心的步骤之一是在反应中心进行的原初反应, PS II 反应中心原初反应的 机理研究已日益成为国际光合作用研究的焦点这一. 1987 年 Nanba 和 Satoh 首次分离纯化 出 PS II 反应中心 D1/D2/Cyt b559 复合物以来, 国际上对 PS II 反应中心的原初电荷分离 (Charge separation)、电荷重组 (Charge recombination)和能量传递过程的动力学性质采用时间 分辨苂光光谱和吸收光谱及烧孔谱 (Hole-burning Spectroscopy) 等技术进行研究, 已取得一些 有意义的结果. 但是, 由于 PS II 反应中心中色素分子的吸收光谱重叠严重, 其吸收光谱在 $675 \mathrm{~nm}$ 处仅有一个吸收峰, 无法选择性地激发原初电子供体 P680, 实验得到的数据比较复杂, 不同实验室得到的动力学数据以及对实验数据的解释, 都存在较大差异, 甚至完全相反 ${ }^{[1]}$.

本文以菠菜叶绿体中的 PS II 颗粒、PS II 核心复合物 (CP47/.CP43/D1/D2/Cyt b559)和 PS II 反应中心复合物 (D1/D2/Cyt b559) 为材料, 用皮秒和飞秒激光技术对光系统 II 反应中心电 荷分离和能量传递的动力学进行研究, 测定出光系统 II 反应中心内部 $\beta$-胡萝卜素和原初电子 供体 P680 之间的能量传递以及 PS II 反应中心原初电荷分离的时间常数, 提出了可能的动力 学模型.

\section{1 材料和方法}

\subsection{PS I 剩粒, PS I 棭心复合物和 PS I 反应中心夏合物的制备}

PSII 颗粒的分离纯化, 参看 Kuwabara 和 Murata ${ }^{[2]}$ 的方法, 样品溶液保存于含 $30 \mathrm{mmol} / \mathrm{L}$ $\mathrm{NaCl}, 50 \mathrm{mmol} / \mathrm{L}$ Tris, $2 \mathrm{mmol}$ Triton X-100 的 $\mathrm{pH} 7.2$ 的 Tris- $\mathrm{HCl}$ 缓冲溶液中, 存贮于液氮中 备用.

2 Ogawa M. Differentiation and proliferation of hematopoietic stem cells. Blood, 1993, 81: 2844

3 Holyoake T L, Alcorn M J. CD34 ${ }^{+}$positive haemopoietic cells; biology and clinical applications. Blood Reviews, 1994; 8; 113

4 Gordon M Y. Human haeomopoietic stem cell assays. Blood Reviews, 1993, 7: 190

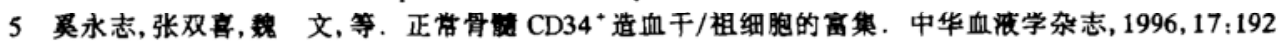

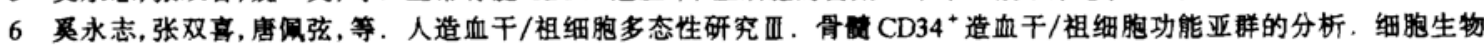
学报, 1996, 29:49

7 本秀菻, 罢永志. 快速富集大量 $\mathrm{CD} 34^{+}$造血千/相细胞的新途径. 解放军医学杂志, 1995, 20:402 